\title{
Standard Model Higgs Boson Searches in ATLAS
}

\author{
J. Vossebeld (on behalf of the ATLAS collaboration) \\ University of Liverpool, Department of Physics, Oliver Lodge Laboratory, Liverpool, L69 7ZE
}

\begin{abstract}
Searches in the ATLAS experiment for a standard model Higgs boson in different decay channels are presented. Cross section upper limits based on all search channels combined are presented over a range of Higgs boson masses, $110<$ $m_{H}<600 \mathrm{GeV}$. The standard model Higgs boson is excluded at $95 \%$ confidence level in the mass ranges $136<m_{H}<196 \mathrm{GeV}$ and $327<m_{H}<443 \mathrm{GeV}$.

PACS: $14.80 . \mathrm{Bn}$
\end{abstract}

\section{INTRODUCTION}

The search for the standard model (SM) Higgs boson is one of the major goals of the Large Hadron Collider. Direct searches at the CERN LEP $e^{+} e^{-}$collider set a lower limit of $114.4 \mathrm{GeV}$ on the Higgs boson mass, $m_{H}$, at $95 \%$ confidence level [1], while searches at the Fermilab Tevatron $p \bar{p}$ collider excluded the additional region $156<m_{H}<177 \mathrm{GeV}[2]$.

Based on over $1 \mathrm{fb}^{-1}$ of $\sqrt{s}=7 \mathrm{TeV}$ proton-proton data collected by the ATLAS experiment up to July 2011, searches for a SM Higgs boson have been performed over a wide range of Higgs masses exploiting different SM Higgs boson production and decay modes. Simulated background and signal events are produced using a wide range of Monte Carlo generators and, where possible, backgrounds are measured directly from data. The cross sections for Higgs boson production and the associated branching fractions used, are compiled in Ref. [3].

\section{SEARCHES IN INDIVIDUAL CHANNELS}

A search for a SM Higgs boson decaying to two photons, updating a previous result described in Ref. [4], is performed in the mass range $110<m_{H}<150 \mathrm{GeV}$. Although the branching fraction for the $H \rightarrow \gamma \gamma$ decay mode is small, this channel has a good sensitivity to a low mass SM Higgs boson, thanks to the excellent mass resolution. Unconverted photons, measured in the ATLAS electro-magnetic calorimeter and converted photons with either one or two tracks reconstructed in ATLAS inner detector are included in the search, which is performed by looking for evidence of a resonant decay in the 2-photon invariant mass, $m_{\gamma \gamma}$, spectrum. Backgrounds to the search occur through genuine photon pair production, Drell-Yan $e^{+} e^{-}$production and events in which one or both photon candidates are fakes, for example from $\pi_{0}$ decays. After stringent quality cuts on the photon candidates, genuine photon pairs form the dominant background contribution. The $m_{\gamma \gamma}$ distribution of the selected candidates (Fig. 1a) is compared to signal and background expectations. No evidence for a resonant decay consistent with a SM Higgs boson is observed.

Although the production of a SM Higgs boson in association with a $W$ or $Z$ boson has a significantly lower cross section than the dominant gluon fusion and vector boson fusion processes, the distinct final state of this production mode allows it to be used in combination with the $H \rightarrow b \bar{b}$ decay mode, favoured for a low mass Higgs boson. A search in this channel [5] requires candidate events to have 2 leptons or 1 lepton and significant missing transverse momentum, consistent with the leptonic decay of a $Z$ or $W$ boson, respectively, and to have 2 jets likely to have originated from $b$-quarks. The search is then performed in the mass range $110<m_{H}<130 \mathrm{GeV}$, by looking for evidence of a SM Higgs boson in the 2-b-jet invariant mass, $m_{b \bar{b}}$, spectra (Fig. $1 \mathrm{~b}$ and $1 \mathrm{c}$ ). The observed candidate distribution in data is consistent with the background expectation.

The decay of the SM Higgs boson to a $W$ boson pair is the dominant decay mode if $m_{H} \gtrsim 135 \mathrm{GeV}$. The decay mode $H \rightarrow W W^{(*)} \rightarrow l v l v$ is exploited to look for the SM Higgs boson in the range $110<m_{H}<240 \mathrm{GeV}$ [6]. Candidate events are required to have an opposite charge lepton pair $(e e, \mu \mu$ or $e \mu)$ and significant missing transverse momentum. Separate search regions are defined for events with 0 or 1 additional jets. After the full selection, the 

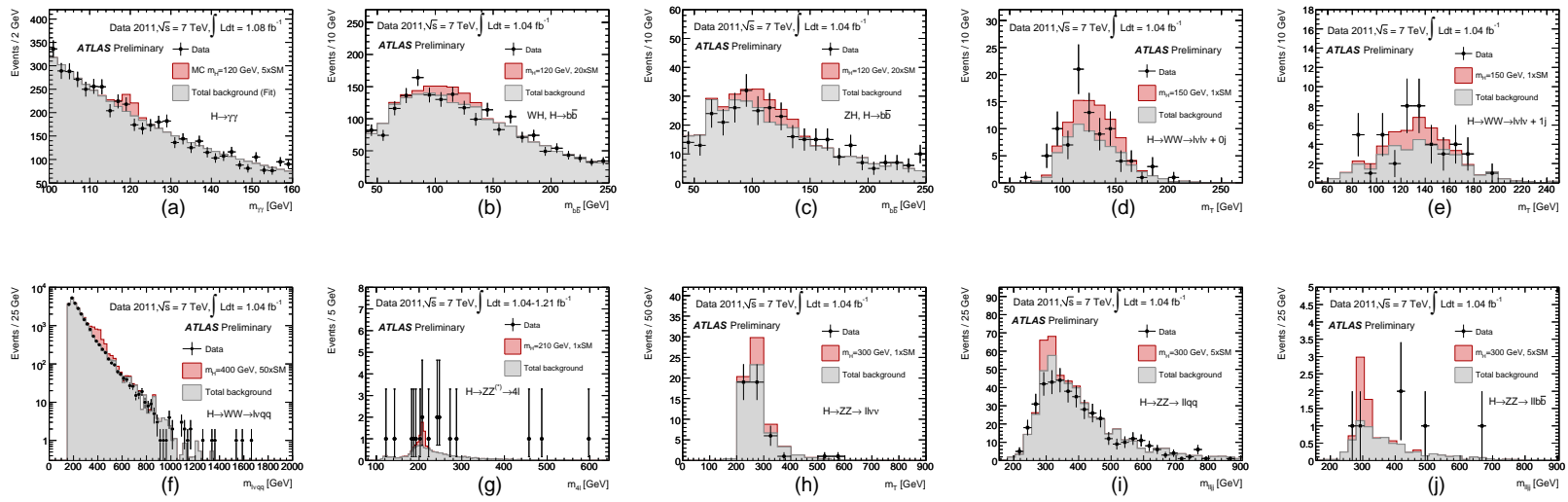

FIGURE 1. The $m_{\gamma \gamma}$ spectrum in the $H \rightarrow \gamma \gamma$ search channel (a), the $m_{b \bar{b}}$ spectra in the $W H$ (b) and $Z H$ (c) search channels, the $m_{T}$ spectra in the $H \rightarrow W W^{(*)} \rightarrow l v l v 0$-jet (d) and 1-jet (e) search channels, the $m_{l v q q}$ spectrum in the $H \rightarrow W W \rightarrow l v q q$ search channel (f), the $m_{4 l}$ spectrum in the $\left.H \rightarrow Z Z^{*}\right) \rightarrow 4 l$ search channel (g), the $m_{T}$ spectrum in $H \rightarrow Z Z \rightarrow l l v v$ channel (h) and the $m_{l l j j}$ spectra in the $H \rightarrow Z Z \rightarrow l l q q$ (i) and $H \rightarrow Z Z \rightarrow l l b b$ (j) channels. The observed distribution for the selected candidates is shown as black data points, while the expected combined background processes are shown as the grey histograms and an example signal expectation as the red histogram.

search is performed based on the number of candidate events in a window applied on the transverse mass distribution, $m_{T}$ (Fig. 1d and 1e). The observed number of candidate events is slightly higher than the background expectation.

The decay mode $H \rightarrow W W \rightarrow l v q q$ is used to look for a SM Higgs boson in the high mass range $240<m_{H}<$ $600 \mathrm{GeV}$, updating a previous search described in Ref. [7]. Events are required to have a lepton and significant missing tranverse momentum consistent a leptonic $W$ boson decay, and 2 jets consistent a hadronic $W$ boson decay. As in the $H \rightarrow W W^{(*)} \rightarrow l v l v$ channel, the search region is separated for candidate events with 0 or 1 additional jets and the search is performed by looking for evidence of a resonance in the invariant mass spectrum of the two $W$ candidates, $m_{l v q q}$ (Fig. 1f), where the missing transverse momentum vector and the $W$ mass are used to estimate the momentum of the unobserved neutrino.

The decay mode $H \rightarrow Z Z^{(*)} \rightarrow 4 l$ is one of the cleanest decay modes in which to search for a SM Higgs boson at the LHC, with only SM ZZ production as a significant background. A search in this channel, updating a previous search described in Ref. [8], is performed by looking for an excess over the SM background in the 4-lepton invariant mass, $m_{4 l}$, spectrum (Fig. 1g) of events, which are required to have two opposite charge lepton pairs, one of which must be consistent with an on-shell $Z$ boson decay. No evidence for a SM Higgs boson is found, although a slight excess of candidate events is observed around $m_{4 l}=250 \mathrm{GeV}$, consistent with a $2 \sigma$ upward fluctuation compared to the expected background.

The $H \rightarrow Z Z \rightarrow l l v v$ and $H \rightarrow Z Z \rightarrow l l q q$ channels have a higher branching fraction than the $H \rightarrow Z Z \rightarrow 4 l$ channel and are used to search for a SM Higgs boson in the high mass range $200<m_{H}<600 \mathrm{GeV}$, updating previous search results described in Ref. [9]. In both channels event are required to have a lepton pair (ee or $\mu \mu)$ consistent with a leptonic $Z$ boson decay. In the $H \rightarrow Z Z \rightarrow l l v v$ channel events are required to also have significant missing transverse momentum and to satisfy further cuts on the opening angle between the leptons and against the presence of jets originating from $b$-quarks. The search is performed by looking for an excess over the background expectations in the transverse mass, $m_{T}$, spectrum based on the lepton pair and the missing transverse momentum (Fig. $1 \mathrm{~h}$ ). In the $H \rightarrow Z Z \rightarrow l l q q$ channel events are required to have 2 jets consistent with the hadronic decay of a $Z$ boson. Two search regions are defined in this channel for events where the 2 jets are identified as likely to have originated from $b$-quarks and for the remaining events. The search is performed by looking for an excess over the background expectations in the invariant mass spectrum of the leptons and the jets, $m_{l l j j}$ (Fig. $1 \mathrm{i}$ and. $1 \mathrm{j}$ ).

\section{CROSS SECTION LIMITS ON THE STANDARD MODEL HIGGS BOSON}

The results in each of the presented search channels are used to set upper limits on the SM Higgs boson production cross section over a range of masses. All limits are based on the $C L_{s}[10]$ confidence level definition and are extracted 
using a frequentist profile likelihood method [11], in which systematic uncertainties are fully accounted for. Cross section upper limits for the SM Higgs boson, based on each of the individual search channels, are shown in Fig. 2(left). The most stringent limits on the SM Higgs boson are those from the $H \rightarrow W W^{(*)} \rightarrow l v l v$ channel in the intermediate $m_{H}$ region and the $H \rightarrow Z Z \rightarrow l l v v$ channel in the high $m_{H}$ region. In the $H \rightarrow W W^{(*)} \rightarrow l v l v$ channel a slight excess of observed candidates leads to an observed limit that is higher than the expected limit. The observed excess would correspond to a $2.5 \sigma$ upward fluctuation of the data compared to the background expectation. The probability to observe such an excess anywhere in the mass range considered, was estimated to be about $8 \%$.

A combination of all channels is performed to obtain the best SM Higgs boson cross section upper limits [12]. Figure 2(right) shows the cross section upper limits on the SM Higgs boson based on all channels combined. The SM Higgs boson is excluded at the $95 \% \mathrm{CL}$ in the mass ranges $136<m_{H}<196 \mathrm{GeV}$ and $327<m_{H}<443 \mathrm{GeV}$.
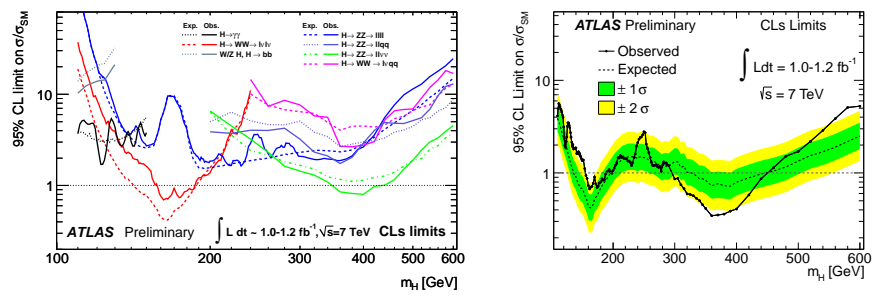

FIGURE 2. Left: Observed (full lines) and expected (dashed or dotted lines) 95\% CL cross section upper limits for the different search channels. Right: Observed (full line) and expected (dotted line) 95\% CL cross section upper limits for the combined search channels. The green and yellow bands indicate variations in the expected limit due to 1 and $2 \sigma$ variations away from the expected background.

\section{CONCLUSION}

Searches for a SM Higgs boson in ATLAS, using different production and decay modes, have been presented. Based on a combination of all search channels, cross section upper limits are placed on the production of the SM Higgs boson in the mass range $110<m_{H}<600 \mathrm{GeV}$. A slight excess of candidates is observed over a range of masses, $130<m_{H}<150 \mathrm{GeV}$, consistent with a $2.5 \sigma$ upward fluctuation in the data with respect to the expected background. The SM Higgs boson is excluded at $95 \% \mathrm{CL}$ in the mass ranges $136<m_{H}<196 \mathrm{GeV}$ and $327<m_{H}<443 \mathrm{GeV}$.

\section{REFERENCES}

1. LEP Working Group for Higgs boson searches, "Search for the Standard Model Higgs boson at LEP", Phys. Lett. B565 61 (2003).

2. The CDF and D0 Collaborations, "Combined CDF and D0 Upper Limits on Standard Model Higgs Boson Production with up to $8.6 \mathrm{fb}-1$ of Data", arXiv:1107.5518 (2011).

3. LHC Higgs Cross Section Working Group, S. Dittmaier, C. Mariotti, G. Passarino and R. Tanaka (Eds.), "Handbook of LHC Higgs Cross Sections: 1. Inclusive Observables", arXiv:1101.0593 (2011).

4. The ATLAS collaboration, "Search for the Higgs Boson in the Diphoton Channel with the ATLAS detector using $209 \mathrm{pb}^{-1} \mathrm{of}^{-}$ 7 TeV Data taken in 2011", ATLAS-CONF-2011-085 (2011).

5. The ATLAS collaboration, "Search for the Standard Model Higgs boson produced in association with a vector boson and decaying to a $b$-quark pair with the ATLAS detector at the LHC", ATLAS-CONF-2011-103 (2011).

6. The ATLAS collaboration, "Search for the Higgs boson in the $H \rightarrow W W \rightarrow l v l v$ decay mode with the ATLAS Detector", ATLAS-CONF-2011-111 (2011).

7. The ATLAS collaboration, "Search for Higgs Boson Production in $p p$ Collisions at $\sqrt{s}=7 \mathrm{TeV}$ using the $H \rightarrow W W \rightarrow l v q q$ Decay Channel and the ATLAS Detector", ATLAS-CONF-2011-052 (2011).

8. The ATLAS collaboration, "Search for the Standard Model Higgs boson in the decay channel $H \rightarrow Z Z \rightarrow 4 l$ with $40 \mathrm{pb}^{-1}$ of $p p$ collisions at $\sqrt{s}=7 \mathrm{TeV}$ ", ATLAS-CONF-2011-048 (2011).

9. The ATLAS collaboration, "Search for a Standard Model Higgs Boson in the Mass Range 200-600 GeV in the Channels $H \rightarrow Z Z \rightarrow l l v v$ and $H \rightarrow Z Z \rightarrow l l q q$ with the ATLAS Detector", ATLAS-CONF-2011-026 (2011).

10. A. Read, "Presentation of search results: The $C L_{S}$ technique", J. Phys. G28 2693-2704 (2002).

11. G. Cowan et al., "Asymptotic formulae for likelihood-based tests of new physics", Eur. Phys. J. C71 1554 (2011).

12. The ATLAS collaboration, "Combination of the Searches for the Higgs Boson in $1 \mathrm{fb}^{-1}$ of Data Taken with the ATLAS Detector at 7 TeV Center-of-Mass Energy", ATLAS-CONF-2011-112 (2011). 